\title{
Sensibilidade ao contraste a grades senoidais de freqüências espaciais baixas em crianças
}

\author{
Contrast sensitivity to sine-wave gratings \\ of low spatial frequencies in children
}

\author{
Natanael Antonio dos SANTOS \\ Valtenice de Cássia Rodrigues de Matos FRANÇA²
}

\begin{abstract}
Resumo
O objetivo deste trabalho foi determinar a função de sensibilidade ao contraste para freqüências espaciais de 0,25; 0,5; 1,0 e 2,0 ciclos por grau em crianças de 4 a 13 anos. Foram estimados limiares de contraste para 60 participantes (50 crianças e 10 adultos jovens), utilizando o método psicofísico da escolha forçada e nível baixo de luminância. Todos os participantes apresentavam acuidade visual normal e se encontravam livres de doenças oculares identificáveis. Os resultados mostraram que a função de sensibilidade ao contraste de crianças de 4-5, 6-7, 8-9, 10-11 e 12-13 anos melhora significativamente com a idade. Os resultados mostraram ainda que a função de sensibilidade ao contraste de crianças de 12-13 anos é semelhante à de adultos jovens (19-22 anos). Estes resultados sugerem que o desenvolvimento da função de sensibilidade ao contraste para grade senoidal em nível baixo de luminância melhora até os 12-13 anos.
\end{abstract}

Unitermos: Criança. Percepção visual. Sensibilidade ao contraste.

\begin{abstract}
The aim of this study was to measure contrast sensitivity function for spatial frequencies of 0.25, 0.5, 1.0 and 2.0 cycles per degree in children between the ages of 4 and 13. We measured the contrast thresholds of 60 participants ( 50 children and 10 young adults) using the psychophysical forced-choice method and low luminance levels. All subjects were free from any identifiable ocular disease and had normal acuity. Results showed that contrast sensitivity function, in children in the 4-5, 6-7, 8-9, 10-11 and 12-13 age ranges, improved significantly with age. The results also showed that, by 12-13 years of age, the contrast sensitivity function was similar to that of a young adult (19-22 years). These results suggest that the development of contrast sensitivity function for sine-wave grating at low luminance levels attains adult-like values by about 12-13 years of age.
\end{abstract}

Uniterms: Child. Visual perception. Contrast sensitivity.

$\boldsymbol{\nabla \nabla v \boldsymbol { v }}$

1 Universidade Federal de Paraíba, Centro de Ciências Humanas, Letras e Artes. Departamento de Psicologia, Laboratório de Percepção, Neurociências e Comportamento. Campus I, Cidade Universitária, 58051-900, João Pessoa, PB, Brasil. Correspondência para/Correspondence to: N.A. SANTOS. E-mail: <natanael.santos@pesquisador.cnpq.br>.

2 Bolsista Fapesp. Universidade de São Paulo, Instituto de Psicologia, Pós-Graduação em Psicologia Experimental. São Paulo, SP, Brasil. Apoio: Conselho Nacional de Desenvolvimento Científico e Tecnológico, nas modalidades Auxílio Individual (processo: 477258/2001-8), Bolsa IC/PIBIC/UFPB/CNPq e Bolsa de Produtividade em Pesquisa (processo: 307182/2003-7). 
A função de sensibilidade ao contraste (FSC) é uma medida que determina a habilidade do sistema visual em detectar objetos (ou padrões do tipo grade senoidal) de freqüências espaciais diferentes em níveis mínimos de contraste. Assim, ela é definida como o inverso da curva de limiar de contraste, ou seja, 1/FSC (Cornsweet, 1970). Neste caso, limiar de contraste é a quantidade mínima de modulação de luminância necessária para detectar um objeto qualquer em função da freqüência espacial. Freqüência espacial (ou grade senoidal) é o número de ciclos (listras claras e escuras) por unidade de espaço, convencionalmente denominado em percepção visual de ciclo por grau de ângulo visual (cpg). O contraste é a relação entre a diferença da luminância máxima e luminância mínima, dividido pela soma das duas.

A FSC é um indicador clássico da percepção visual e um dos instrumentos mais importantes na análise teórica e clínica de estruturas da visão (Adams \& Courage, 2002; Montés-Micó \& Ferrer-Blasco, 2001). Ela descreve o desempenho do sistema visual em níveis diferentes de contrastes, fornecendo uma das descrições mais completas do sistema visual humano (Adams \& Courage, 2002; Wilson, Levi, Maffei, Rovamo \& De Valois, 1990). A FSC tem sido uma das ferramentas mais utilizadas para detectar e avaliar alterações nas vias sensoriais visuais produzidas por vários transtornos, como: esquizofrenia (Slaghuis \& Thompson, 2003), síndrome de Down (Suttle \& Turner, 2004), ambliopia (Polat, Sagi \& Norcia, 1997), catarata (Elliott \& Situ, 1998), intoxicação por mercúrio (Canto-Pereira et al., 2005; Ventura et al., 2005) e doenças de Alzheimer e de Parkinson (Akutsu \& Legge, 1995; Bour \& Apkarian, 1996; Elliott \& Situ, 1998; Polat et al., 1997; Vleugels, van Nunen, Lafosse, Ketelaer \& Vandenbussche, 1998), entre outros.

Sensibilidade ao contraste e desenvolvimento visual

Tradicionalmente, a FSC tem sido utilizada para avaliar o desenvolvimento do sistema visual de crianças desde a década 70, a partir dos estudos de Atkinson, Braddick e Braddick (1974) e Atkinson, Braddick e Moar (1977). Entretanto, a maioria das pesquisas foi realizada com bebês e com as técnicas de potencial visual evocado e olhar preferencial (Allen, Tyler \& Norcia, 1996; Atkinson et al., 1974; Atkinson et al., 1977; Cannon JR, 1983. Peterzell, Werner \& Kaplan, 1995). Estes estudos
}

logo destacaram que a FSC é muito pobre ao nascimento, melhorando rapidamente durante os seis primeiros meses. Relataram, ainda, que o processo de maturação da FSC se estenderia além da infância inicial e do período pré-escolar (3-5 anos). Neste contexto, surgiram estudos visando determinar o período em que a FSC da criança alcança o desempenho ou o perfil do adulto (Adams \& Courage, 2002; Benedek, Benedek, Kéri \& Janáky, 2003; Bradley \& Freeman, 1982; Ellemberg, Lewis, Liu, \& Maurer, 1999). Por exemplo, Bradley e Freeman (1982) mediram a FSC de crianças de 2 a 16 anos, utilizando uma variação da técnica da escolha forçada da escada (forced-choice staircase technique) de duas alternativas e grades senoidais com luminância média $250 \mathrm{~cd} / \mathrm{m}^{2}$. Os resultados indicaram que a FSC melhora com a idade e que as crianças atingem os níveis de sensibilidade ao contraste dos adultos por volta dos 8 anos. Estes pesquisadores demonstraram, ainda, que o método psicofísico foi eficiente para medir a FSC de crianças a partir dos 3,5 anos.

Por sua vez, Ellemberg et al. (1999) mediram a FSC de crianças de 4 a 7 anos e adultos, utilizando o método psicofísico dos limites e grades senoidais com luminância média de $9 \mathrm{~cd} / \mathrm{m}^{2}$. Eles encontraram que a FSC melhora com o aumento da idade e que o desempenho das crianças de 7 anos foi semelhante ao dos adultos.

Por outro lado, Adams e Courage (2002) mediram a FSC de crianças de 1 mês a 9 anos de idade, utilizando o método psicofísico do olhar preferencial e grades senoidais impressas em cartões com luminância média de $70 \mathrm{~cd} / \mathrm{m}^{2}$. Os resultados mostraram que a sensibilidade ao contraste das crianças desenvolve-se rapidamente até os três anos de idade; após isso, a sensibilidade ao contraste continua desenvolvendo-se mais lentamente, atingindo, finalmente, níveis de sensibilidade ao contraste dos adultos por volta dos 9 anos.

Finalmente, Benedek et al. (2003) mediram a FSC de crianças e adolescentes de 5 a 14 anos, utilizando o método psicofísico do ajustamento e grades senoidais com dois níveis de luminância média $(0,09$ e 9,00cd/m²). Os dados mostraram que a FSC melhora gradativamente com a idade, atingindo a sensibilidade do adulto por volta dos 11-12 anos.

Em linhas gerais, os estudos encontrados na literatura mostram que a FSC para grades senoidais melhora gradativamente com a idade e que a identificação do 
período de maturação do sistema visual da criança depende das condições de visualização do método psicofísico empregado.

Neste estudo, mediu-se a FSC para grades senoidais com freqüências espaciais na faixa de 0,25 a 2,00cpg, em crianças de 4 a 13 anos, divididas em cinco grupos (4-5, 6-7, 8-9, 10-11 e 12-13 anos). Mediu-se ainda a FSC de adultos jovens (19-22 anos) para efeito de comparação. As medições foram feitas com o método psicofísico da escolha forçada em nível baixo de luminância (luminância média de $0,7 \mathrm{~cd} / \mathrm{m}^{2}$ ). O objetivo principal foi caracterizar a existência de relações entre o desenvolvimento visual e as estimativas da FSC para grades senoidais verticais, com o método psicofísico da escolha forçada em nível baixo de luminância. As pesquisas que determinaram a FSC de crianças, em sua maioria, utilizaram grades senoidais verticais com níveis altos de luminância (Adams \& Courage, 2002; Bradley \& Freeman, 1982; Ellemberg et al., 1999). Além disto, não foi encontrado na literatura nenhum estudo relacionando medidas de sensibilidade ao contraste e o desenvolvimento a partir da idade pré-escolar, com o método psicofísico da escolha forçada.

\section{Método}

\section{Participantes}

Participaram do estudo 60 voluntários, 50 crianças ( 28 do sexo feminino e 22 do sexo masculino) e 10 adultos (seis do sexo feminino e quatro do sexo masculino). Os mesmos foram distribuídos em seis grupos etários com dez participantes [4-5 anos ( $n=10$; Média- $M=4,95$; desvio-padrão- $D P=0,65), 6-7$ anos ( $n=10$; $M=6,87 ; D P=0,68), 8-9$ anos $(n=10 ; M=8,74 ; D P=0,77)$, 10-11 anos ( $n=10 ; M=10,73 ; D P=0,46), 12-13$ anos $(n=10$; $M=11,95 ; D P=0,57$ ) e adultos jovens de 19-22 anos ( $n=10$; $M=21,32$ anos; $D P=0,91$ )]. Todos apresentavam acuidade visual normal ou corrigida, e não tinham história de doença ocular ou neurológica. A participação na pesquisa ocorreu mediante assinatura de Termo de Consentimento Livre e Esclarecido, conforme a Resolução n¹96/96 do Conselho Nacional de Saúde (Ministério da Saúde), que trata das diretrizes e normas de pesquisas envolvendo seres humanos. No caso das crianças, o termo foi assinado pelo responsável. O projeto foi aprovado pelo comitê de Ética do Centro de Ciências da Saúde da Universidade Federal da Paraíba.

\section{Equipamentos}

Os estímulos foram gerados em um monitor de vídeo Clinton Medical monocromático de 21 polegadas, digital e de alta resolução, controlado por um microcomputador. Uma cadeira foi fixada a $150 \mathrm{~cm}$ da tela do monitor de vídeo. A luminância média dos estímulos foi de $0,7 \mathrm{~cd} / \mathrm{m}^{2}$, ajustada por um fotômetro do tipo Spot Metter, com precisão de um grau, Asahi Pentax. A luminância mínima foi de $0,5 \mathrm{~cd} / \mathrm{m}^{2}$ e a máxima de $0,9 \mathrm{~cd} / \mathrm{m}^{2}$. O ambiente do laboratório era cinza para melhor controle da luminância média $\left(3,5 \mathrm{~cd} / \mathrm{m}^{2}\right)$.

Foram utilizados estímulos do tipo grade senoidal vertical, com freqüências espaciais de 0,25; 0,50; 1,00 e 2,00 cpg. Estes eram circulares, com um diâmetro de 7 graus de ângulo visual a $150 \mathrm{~cm}$ de distância da tela, e foram gerados em tons de cinza e apresentados em tempo real no monitor (Figura 1).

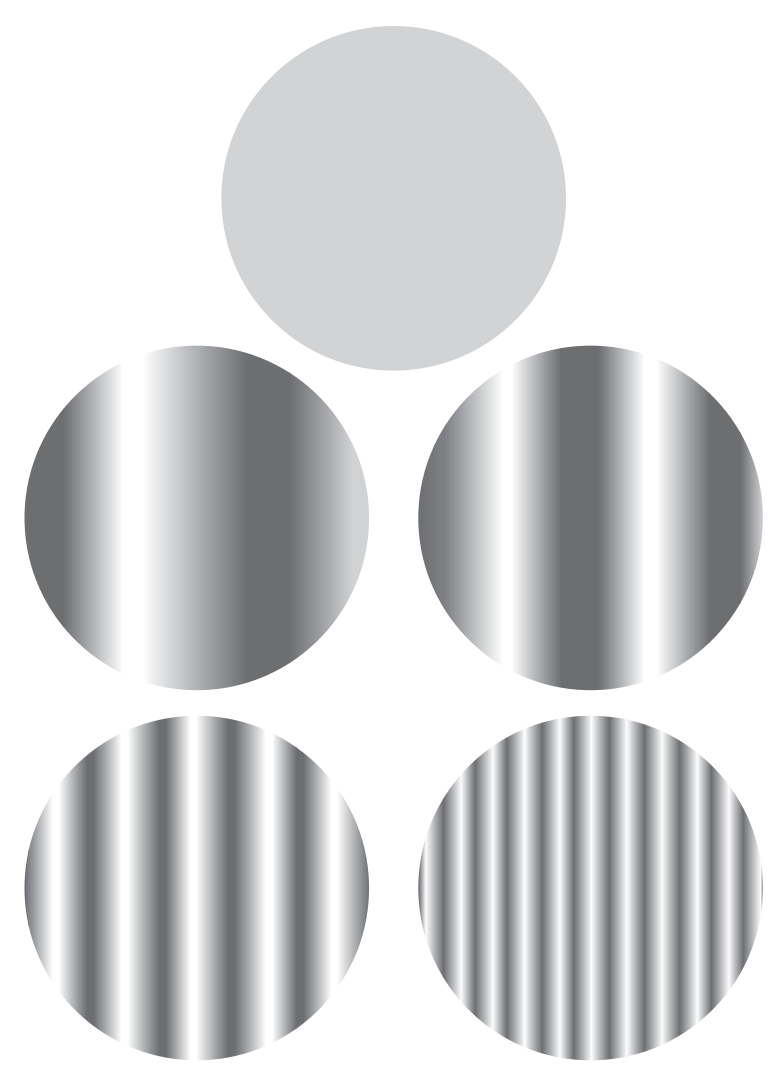

Figura 1. Exemplos de estímulo neutro (acima) e estímulos de freqüências espaciais (no centro, da esquerda para a direita, 0,25 e 0,50 cpg, e embaixo, da esquerda para direita, 1,00 e 2,00 cpg). Estímulos originalmente calibrados para serem vistos a $150 \mathrm{~cm}$ de distância. 


\section{Procedimentos}

As estimativas foram realizadas com o método psicofísico da escolha forçada (Santos, Nogueira \& Simas, 2005; Santos, Oliveira, Nogueira \& Simas, 2006; Wetherill \& Levitt, 1965). Este método se baseia no cálculo da probabilidade de acertos consecutivos por parte do participante, ou seja, em cerca de 100 apresentações de escolhas entre os dois estímulos, a freqüência espacial (estímulo de teste) foi percebida, por exemplo, em $79,00 \%$ das vezes pelo voluntário. O procedimento para medir o limiar para cada freqüência consistiu na apresentação sucessiva simples do par de estímulos, no qual um deles era o estímulo teste, que deveria ser identificado pelo participante. O outro estímulo (estímulo neutro) foi sempre um padrão homogêneo com luminância média de $0,7 \mathrm{~cd} / \mathrm{m}^{2}$. O critério adotado para variar o contraste de cada freqüência espacial testada foi o de três acertos consecutivos para decrescer uma unidade, e um erro para acrescer a mesma unidade $(0,08 \%)$.

Durante cada sessão experimental foi apresentada uma seqüência de estímulos iniciada com um sinal sonoro, acompanhado imediatamente pela apresentação do primeiro estímulo por 2 segundos, seguido de um intervalo entre estímulos de 1 segundo e pela apresentação do segundo estímulo por 2 segundos, e da resposta do participante. A ordem de apresentação dos estímulos era aleatória. Os participantes foram instruídos a pressionar o botão do lado esquerdo do mouse quando a grade senoidal (ou freqüência espacial) fosse apresentada em primeiro lugar, e o botão do lado direito quando fosse apresentada em segundo lugar, isto é, após o estímulo neutro. Se a resposta do voluntário fosse correta, era seguida por outro sinal sonoro e um intervalo de 3 segundos para a seqüência se repetir. O sinal sonoro que indicava o início da apresentação do par de estímulos e o que indicava a escolha correta eram diferentes. A duração da sessão experimental variou entre 5 e 10 minutos, dependendo dos erros e acertos do participante, até proporcionarem um total de cinco reversões (ou cinco valores de contraste), conforme requerido para o final automático da mesma.

Cada uma das FSC foi estimada pelo menos duas vezes (duas sessões experimentais), em dias diferentes, por cada um dos participantes. Em média, vinte curvas foram medidas para cada grupo de voluntários, totalizando 80 sessões experimentais. Todas as estimativas foram medidas à distância de $150 \mathrm{~cm}$, com visão binocular. Importante mencionar que os experimentos só começaram quando o experimentador certificou-se que os participantes entenderam e responderam conforme as instruções.

Após cada sessão, o programa produziu uma folha de resultados com a situação experimental e os cinco valores de contraste. Esses foram organizados em planilhas por faixa etária e por freqüência espacial, produzindo uma grande média que foi utilizada como estimativa do limiar de contraste (ou sensibilidade ao contraste).

\section{Resultados}

Os dados foram tratados com ANOVA (Repeated Measures ANOVA) com dois fatores (freqüência espacial e idade), seguida pelo teste post-hoc de Tukey HSD para comparações entre idades e freqüências espaciais. As análises permitiram observar um efeito principal significante de idade, $\mathrm{F}_{(5,2790)}=271,95(p<0,001)$, um efeito principal significante de freqüência espacial, $F_{(3,2790)}=1480,32$ $(p<0,001)$, e uma interação significante de idade versus freqüência espacial, $F_{(15,2790)}=341,33(p<0,001)$. As análises com o teste de Tukey mostraram diferenças significativas entre a FSC de crianças de 4-5 e 6-7 anos em todas as freqüências $(p<0,001)$; 6-7 e 8-9 anos nas freqüências de 1,00 cpg $(p<0,001)$ e 2,00cpg $(p<0,010)$; 8-9 e 10-11 anos nas freqüências de $0,25 \mathrm{cpg}(p<0,01), 0,50 \mathrm{cpg}(p<0,001)$ e $1,00 \mathrm{cpg}(p<0,01)$, e não foram encontradas diferenças significativas entre as crianças de 10-11 e 12-13 anos. Já para as crianças de 10-11 anos e adultos, foram encontradas diferenças significativas em todas as freqüências $(p<0,001)$. Entretanto, não houve diferença significativa entre a FSC de crianças de 12-13 anos e adultos em nenhuma das freqüências testadas.

A Figura 2 mostra a FSC média para cada uma das seis faixas etárias (crianças de 4-5, 6-7, 8-9, 10-11, 12-13 anos e adultos jovens). Os limiares de contraste são apresentados em função da freqüência espacial. A FSC é o inverso do limiar de contraste (1/FSC). Em outras palavras, quanto menor o limiar de contraste, maior a sensibilidade do sistema visual humano e vice-versa. Assim, os menores valores de limiares correspondem aos maiores valores de sensibilidade ao contraste. 


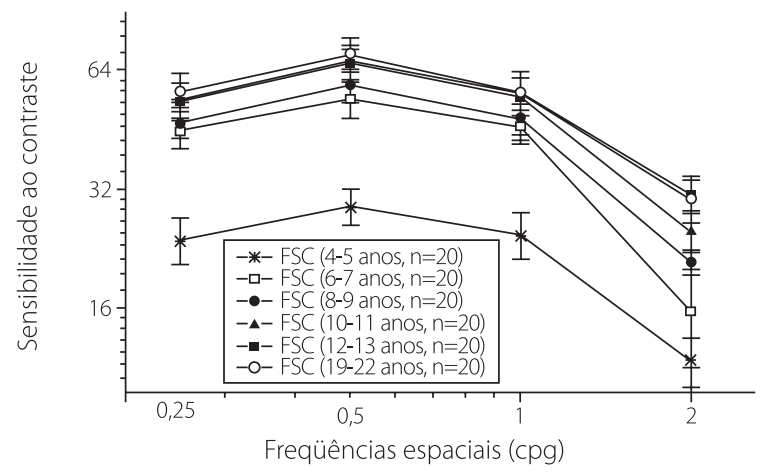

Figura 2. Curvas de sensibilidade ao contraste de crianças de 4-5, 6-7, 8-9, 10-11, 12-13 anos e adultos jovens (19-22 anos) para freqüências espaciais (FSC). O n representa o número de curvas mensuradas para cada faixa etária. As linhas verticais mostram o erro padrão da média para cada freqüência $(0,25 ; 0,50 ; 1,00$ e 2,00 cpg).

A sensibilidade máxima ocorreu na freqüência espacial de 0,5cpg para todas as idades (Figura 2). Entretanto, a sensibilidade ao contraste das crianças de 4-5 anos na freqüência de 0,5cpg foi da ordem de 1,57 vezes menor do que a das crianças de 6-7 anos. Já a sensibilidade das crianças de 6-7 anos foi da ordem de 1,13 vezes menor do que a das crianças de 8-9 anos que, por sua vez, foi da ordem de 1,22 vezes menor do que a das crianças de 10-11 anos que, finalmente, foi da ordem de 1,04 vezes menor do que a das crianças de 12-13 anos. Por outro lado, o limiar de contraste das crianças de 12-13 anos foi da ordem de 1,04 vezes menor do que a dos adultos na freqüência de 0,5cpg.

No geral, estes resultados mostram que a FSC melhora gradativamente e de forma significante com o aumento da idade. Os resultados mostram ainda que, embora não exista diferença estatística entre as crianças de 10-11 e 12-13 anos, a FSC das crianças só atinge o padrão do adulto por volta 12-13 anos, quando a FSC das crianças é estatisticamente semelhante à dos adultos.

\section{Discussão}

Os resultados mostraram que o método psicofísico da escolha forçada, tradicionalmente utilizado em pesquisas com adultos, pode ser utilizado de forma confiável para medir a curva de sensibilidade ao contraste de freqüências espaciais verticais de crianças a partir dos 4-5 anos. A Figura 2 demonstra que a FSC das crianças de 4-5 anos apresenta um perfil geral muito semelhante às curvas de sensibilidade de crianças de 67, 8-9, 10-11, 12-13 anos e adultos jovens (19-22 anos). A diferença entre a FSC das crianças de 4-5 anos e a FSC das outras faixas etárias pode ser relacionada ao domínio da sensibilidade ao contraste, que aumenta gradativamente com a idade. Este dado é importante porque a medição da FSC com método psicofísico geralmente tem uso limitado quando lida com crianças em idades pré-escolares. Tal fato é tão relevante que os estudos que mediram a FSC de crianças pré-escolares para grades senoidais fizeram adaptações no aparato e procedimento e, ainda assim, só conseguiram medir a FSC a partir dos 3 anos (Bradley \& Freeman, 1982; Richman \& Lyons, 1994). Por exemplo, Richman e Lyons mediram a FSC de crianças a partir dos três anos utilizando um procedimento simples, no qual os estímulos eram apresentados em cartões (Vistech Chart).

Um procedimento semelhante foi utilizado por Bradley e Freeman (1982), que conseguiram medir a FSC de crianças a partir de 3,5 anos. Ou seja, em ambos os estudos, a criança deveria apenas olhar ou apontar para o lado ou direção que continha a grade. Estes aspectos podem explicar porque esses autores conseguiram mensurar a FSC com método psicofísico a partir dos 3 anos, um vez que os métodos psicofísicos geralmente utilizados com adultos nem sempre são efetivos quando testam crianças pré-escolares (Bradley \&Freeman, 1982; Richman \& Lyons, 1994).

Por outro lado, retornando ao objetivo principal deste estudo, que foi procurar a existência de relações entre o desenvolvimento visual de crianças de 4 a 13 anos e as estimativas de curva de sensibilidade ao contraste para grades senoidais verticais com o método psicofísico da escolha forçada em nível baixo de luminância, os resultados mostraram que a faixa de maior sensibilidade ocorreu na freqüência espacial de 0,5 cpg para todas as idades. Isto significa que, embora a FSC melhore com o aumento da idade, a faixa de freqüência espacial em que o sistema visual é mais sensível continua em 0,5cpg. Entretanto, os resultados demonstram que o processo de maturação da FSC ocorre de forma mais rápida entre os 4-5 e 6-7 anos, pois o desempenho das 
crianças de 4-5 em relação ao das crianças de 6-7 anos foi estatisticamente significativo em todas as freqüências $(p<0,001)$ (Figura 2).

A Figura 2 mostra que, após os 6-7 anos, o processo de desenvolvimento da FSC vai se tornando mais gradual (i.e, as diferenças entre as faixas etárias são menores), exceto na freqüência de 2cpg, na qual mudanças significativas foram encontradas entre todas as idades, até os 10-11 anos ( $p<0,001)$. Nas outras freqüências, a partir dos 6-7 anos, observa-se uma melhora gradativa na FSC até alcançar o padrão do adulto, inclusive na faixa de máxima sensibilidade (0,5cpg). Aumento na FSC relacionado ao desenvolvimento já era esperado, pois vários estudos com grades senoidais verticais em níveis altos de luminância (ou contraste) relataram dados nesta direção, com métodos psicofísicos diferentes (Adams \& Courage, 2002; Arundale, 1978; Benedek et al., 2003; Bradley \& Freeman, 1982; Ellemberg et al., 1999).

Diferente dos estudos anteriores que relataram que a FSC das crianças melhora até os 7-9 anos, quando atinge o perfil dos adultos, os resultados deste estudo mostram que a FSC de crianças para grade senoidal só atinge o perfil do adulto por volta dos 12-13 anos. Estes resultados são semelhantes aos dados de Benedek et al. (2003), que encontraram que a FSC de crianças se desenvolve até os 11-12 anos, quando, então, torna-se semeIhante à do adulto. Entretanto, Benedek et al. (2003) também utilizaram nível baixo de luminância $\left(0,09 \mathrm{~cd} / \mathrm{m}^{2}\right)$.

A luminância é importante porque a FSC depende das condições de visualização. Por exemplo, os resultados deste estudo com luminância média baixa $\left(0,7 \mathrm{~cd} / \mathrm{m}^{2}\right)$, comparados aos dados encontrados na literatura com luminância média mais alta (Adams \& Courage, 2002; Bradley \& Freeman, 1982; Ellemberg et al., 1999), mostram que a utilização de luminância baixa desloca a zona de máxima sensibilidade para a esquerda da FSC (i.e, para a freqüência de 0,5cpg). No entanto, em níveis de luminância alta, a faixa de máxima sensibilidade ocorre por volta de 3,0 a 5,0cpg. Além disto, em níveis baixos de luminância, o sistema visual humano possivelmente não detecta freqüências espaciais acima de 3,0cpg (Benedek et al., 2003).

Por isto, este trabalho se limitou a investigar freqüências espaciais mais baixas. $O$ deslocamento da 182 sensibilidade máxima para freqüências mais baixas (p.ex., 0,5cpg) pode estar relacionado ao funcionamento da via visual magnocelular, que é especializada no processamento de freqüências espaciais baixas, em níveis baixos de luminância (Benedek et al., 2003; Ellemberg et al., 1999). Em outras palavras, o processamento visual da forma e contraste envolve pelo menos dois sistemas: a via visual parvocelular, que é especializada no processamento de freqüências espaciais médias e altas ou detalhes finos e opera em níveis altos ou fotópicos de luminância, e a via visual magnocelular, que é especializada no processamento de freqüências espaciais baixas e opera em níveis baixos ou escotópicos de luminância (Benedek et al., 2003; Ellemberg et al., 1999).

Neste contexto, os resultados deste estudo reforçam a hipótese de Benedek et al. de que o processo de maturação da FSC é mais lento em nível baixo de luminância. Outras informações quanto à influência neuronal no desenvolvimento da FSC podem ser encontradas na literatura (Banks \& Bennett, 1988; Brown, Dobson \& Maier, 1987; Ellemberg, Lewis, Maurer \& Brent, 2000; Hickey, 1977; Kiorpes \& Movshon, 1998; Youdelis \& Hendrickson, 1986; van Sluyters, Atkinson, Held, Hoffman \& Shatz, 1990; Wilson, 1988). Inclusive, estes estudos mostram que a maturação da FSC parece diretamente relacionada ao desenvolvimento morfológico e neurofisiológico, que segue uma escala de tempo contínua e específica durante a qual o sistema nervoso se organiza e se estrutura.

É provável que o nível de luminância explique, em parte, os resultados diferentes encontrados na literatura, pois os trabalhos geralmente utilizam métodos distintos, impedindo uma comparação direta. Embora os métodos psicofísicos até então utilizados para mensurar a FSC de crianças tenham sido bem sucedidos, os mesmos não geraram resultados totalmente semelhantes, pois existem, entre outros aspectos, dificuldades com a variabilidade na estimação da sensibilidade ao contraste (Billock \& Harding, 1996). Além do mais, entende-se que qualquer método simples pode ter limitações, até porque o mesmo depende de interações espaço-temporais que são diferentes em cada caso (Benedek et al., 2003; Ellemberg et al., 1999). Neste sentido, estes fatores podem justificar a falta de consenso quanto ao processo de maturação da FSC ou ao período em que a criança atinge o desempenho do adulto na maioria das pesquisas. 
Em termos gerais, os resultados envolvendo o desenvolvimento da FSC são consistentes com a hipótese de que a caracterização da FSC deve levar em consideração as condições de apresentação e visualização dos estímulos e o método psicofísico utilizado (Billock \& Harding, 1996; Georgeson \& Sullivan, 1975; Wilson \& Wilkinson, 1997). Principalmente, quando se visa especificar que tipo de objeto (ou estímulo) uma criança de uma determinada idade pode detectar, ou quando o intuito é descrever o desenvolvimento e funcionamento de mecanismos neurofisiológicos básicos relacionados às funções visuais ou, ainda, determinar parâmetros para pesquisa teórica e clínica. É nesta perspectiva que se prosseguirá com as investigações.

\section{Referências}

Adams, R. J., \& Courage, M. L. (2002). Using a single test to measure human contrast sensitivity from early childhood to maturity. Vision Research, 42 (9), 1205-1210.

Akutsu, H., \& Legge, G. E. (1995). Discrimination of compound gratings: spatial-frequency channels or local features? Vision Research, 35 (19), 2685-2695.

Allen, D., Tyler, C. W., \& Norcia, A. M. (1996). Development of grating acuity and contrast sensitivity in the central and peripheral visual field of the human infant. Vision Research, 36 (13), 1945-1953.

Arundale, K. (1978). An investigation into the variation of human contrast sensitivity with age and ocular pathology. British Journal of Ophthalmology, 62 (4), 213-215.

Atkinson, J., Braddick, O., \& Braddick, F. (1974). Acuity and contrast sensitivity of infant vision. Nature, 247 (5440), 403-404.

Atkinson, J., Braddick, O., \& Moar, K. (1977). Contrast sensitivity of the human infant for moving and static patterns. Vision Research, 17 (9), 1045.1047.

Banks, M. S., \& Bennett, P. J. (1988). Optical and photoreceptor immaturities limit the spatial and chromatic vision of human neonates. Journal of the Optical Society of America A, 5 (12), 2059-2079.

Benedek, G., Benedek, K., Kéri, S., \& Janáky, M. (2003). The scotopic low-frequency spatial contrast sensitivity develops in children between the ages of 5 and 14 years. Neuroscience Letters, 345 (3), 161-164.

Billock, V., \& Harding, T. (1996). Evidence of spatial and temporal channels in the correlational structure of human spatialtemporal contrast sensitivity. Journal of Physiology, 490 (2), 509-517.

Bour, L. J., \& Apkarian, P. (1996). Selective broad-band spatial frequency loss in contrast sensitivity functions. Investigative Ophthalmology \& Visual Science, 37 (12), 2475-2484.
Bradley, A., \& Freeman, R. D. (1982). Contrast sensitivity in children. Vision Research, 22 (8), 953-959.

Brown, A. M., Dobson, V., \& Maier, J. (1987). Visual acuity of human infants at scotopic, mesopic, and photopic luminances. Vision Research, 27 (10), 1845-1858.

Cannon JR, M. W. (1983). Contrast sensitivity: Psychophysical and evoked potential methods compared. Vision Research, 23 (1), 87-95.

Canto-Pereira, L. H. M., Lago, M., Costa, M. F., Rodrigues, A. R., Saito, C. A., Silveira, L. C. L., \& Ventura, D. F. (2005). Visual impairment on dentists related to occupational mercury exposure. Environmental Toxicology and Pharmacology, 19 (3), 517-522.

Cornsweet, T. N. (1970). Visual perception. New York: Academic Press.

Ellemberg, D., Lewis, T. L., Liu, C. H., \& Maurer, D. (1999). Development of spatial and temporal vision during childhood. Vision Research, 39 (14), 2325-2333.

Ellemberg, D., Lewis, T. L., Maurer, D., \& Brent, H. P. (2000). Influence of monocular deprivation during infancy on the later development of spatial and temporal vision. Vision Research, 40 (23), 3283-3295.

Elliott, D. B., \& Situ, P. (1998). Visual acuity versus letter contrast sensitivity in early cataract. Vision Research, 38 (13), 2047-2052.

Georgeson, M. A., \& Sullivan, G. D. (1975). Contrast Constancy: deblurring in human vision by spatial frequency channels. Journal of Physiology, 252 (3), 627-656.

Hickey, T. L. (1977). Postnatal development of the human lateral geniculate nucleus: Relationship to a critical period for the visual system. Science, 198 (4319), 836-838.

Kiorpes, L., \& Movshon, J. A. (1998). Peripheral and central factors limiting the development of contrast sensitivity in macaque monkeys. Vision Research, 38 (1), 61-70.

Montés-Micó, R., \& Ferrer-Blasco, T. (2001). Contrast sensitivity function in children: Normalized notation for the assessment and diagnosis of diseases. Documenta Ophthalmologica, 103 (3), 175-186.

Peterzell, D. H., Werner, J., \& Kaplan, P. S. (1995). Individual differences in contrast sensitivity functions: Iongitudinal study of 4-, 6- and 8-month-old human infants. Vision Research, 35 (7), 961-979.

Polat, U., Sagi, D., \& Norcia, A. M. (1997). Abnormal long-range spatial interactions in amblyopia. Vision Research, 37 (6), 737-744.

Richman, J. E., \& Lyons, S. (1994). A forced choice procedure for evaluation of contrast sensitivity function in preschool children. Journal of the American Optometric Association, 65 (12), 859-864.

Santos, N. A., Nogueira, R. M. T. B. L., \& Simas, M. L. B. (2005). Processamento visual da forma: evidências para canais múltiplos de freqüências angulares em humanos. Psicologia Reflexão \& Crítica, 18 (1), 98-103.

Santos, N. A., Oliveira, A. B., Nogueira, R. M. T. B. L., \& Simas, M. L. B. (2006). Mesopic radial frequency contrast 
sensitivity function for young and older adults. Brazilian Journal of Medical and Biological Research, 39 (6), 791-794.

Slaghuis, W. L., \& Thompson, A. K. (2003). The effect of peripheral visual motion on focal contrast sensitivity in positive- and negative-symptom schizophrenia. Neuropsychologia, 41 (8), 968-980.

Suttle, C. M., \& Turner, A. M. (2004). Transient pattern visual evoked potentials in children with Down's syndrome. Ophthalmic and Physiological Optics, 24 (2), 91-99.

van Sluyters, R. C., Atkinson, M. S., Held, R. M., Hoffman, K., \& Shatz, C. J. (1990). the development of vision and visual perception. In S. W. Spillmann \& J. S. Werner (Orgs.), The neurophysiological foundation (pp.349-379). New York: Academic Press.

Ventura, D. F., Simões, A. L., Tomaz, S., Costa, M. F., Lago, M., Costa, M. T. V., Canto-Pereira, L. H. M., Souza, J. M., Faria, M. A. M., \& Silveira, L. C. L. (2005). Colour vision and contrast sensitivity losses of mercury intoxicated industry in Brazil. Environmental Toxicology and Pharmacology, 19 (3), 523-529.

Vleugels, L., van Nunen, A., Lafosse, C., Ketelaer, P., \& Vandenbussche, E. (1998). Temporal and spatial resolution in foveal vision of multiple sclerosis patients. Vision Research, 38 (19), 2987-2997.
Wetherill, G. B., \& Levitt, H. (1965). Sequential estimation of points on a psychometric function. The British Journal of Mathematical and Statistical Psychology, 48 (1), 1-10.

Wilson, H. R. (1988). Development of spatiotemporal mechanisms in infant vision. Vision Research, 28 (5), 611-628.

Wilson, H. R., Levi, D., Maffei, L., Rovamo, J., \& De Valois, R. (1990). The perception of form: Retina to striate cortex. In S. W. Spillmann \& J. S. Werner (Orgs.), Visual perception: theneurophysiological foundation (pp.231-271). New York: Academic Press.

Wilson, H. R., \& Wilkinson, F. (1997). Evolving concepts of spatial channels in vision: from independence to nonlinear interactions. Perception, 26 (8), 939-960.

Yuodelis, C., \& Hendrickson, A. (1986). A qualitative and quantitative analysis of the human fovea during development. Vision Research, 26 (6), 847-855.

Recebido em: 4/12/2006

Versão final reapresentada em: 4/5/2007

Aprovado em: 3/7/2007 